\title{
Research on Recommendation Model of Mobile Commerce based on Personalized Context
}

\author{
Du Wei ${ }^{1}$, Gao Chang-yuan ${ }^{1,2 *}$ and Zhang Shu-chen ${ }^{1,2}$ \\ ${ }^{1}$ College of Management, Harbin University of Science and Technology, Harbin, \\ Heilongjiang, 150040, China \\ ${ }^{2}$ High-tech Industrial Development Research Center, Harbin University of Science \\ and Technology, Heilongjiang, 150040, China \\ "gaocy2002@126.com
}

\begin{abstract}
Most of the overall context for the user's overall recommendation for mobile commerce research context is based on the object of study, the lack of in-depth analysis of each user a personalized context, lead to the decline in the quality of the recommendation, the user experience is insufficient. In this paper, factors affecting the user immediate information needs of the context elements are classified, put forward a kind of recommendation model of mobile commerce which is integrated into the user's personalized context elements. In this model, the current context by the user and historical data to calculate the score for each user immediate impact on the information needs of the largest elements of the $K$ context, this configuration personalized user profile, and improve the existing conditions as an input-based context multidimensional information recommendation algorithm and program recommendations. By Movie lens and Book-Crossing the model data set Improved algorithms and other three algorithms were compared, results showed that: high prediction accuracy of the model can effectively improve the user experience satisfaction, more effective solution to mobile personalized commerce environment recommendation problem.
\end{abstract}

Keyword: e-commerce; context element; personalized context; multidimensional information recommendation

\section{Introduction}

In recent years, with the development of mobile communication technology and the popularity of smart mobile devices, users desperately want to be able to timely, convenient and effective access to meet their demand for personalized information and services from the mobile Internet on the move, e-commerce start into the various mobile terminals, mobile e-commerce came into being and developed rapidly. However, due to the growing information technology and the rapid development of information content, combined with mobile devices ability to process information is still limited, making it increasingly difficult to get valuable information obtained through the mobile network, resulting in a mobile network and mobile users order conversion rate experience severely affected. In order to effectively solve the" mobile information" overload problem, move recommendation system into the people's vision to become an effective means to alleviate this problem, received extensive attention.

Currently, the information needs for mobile business users with a strong dependence of the characteristics of the context, scholars have begun working on the problem in a mobile environment combined with recommended user profile information. Vico, who proposed a two-stage moving context recommended model, first consider the state of weighting user

${ }^{*}$ Corresponding Author 
context weight, if weight reaches a certain threshold, recycling or other collaborative filtering recommendation algorithm to recommend [1]. Jeong, who will take into account the context information recommendation model and genetic algorithm combined with collaborative filtering algorithm to recommend ways to effectively improve the recommendation quality[2].Yuan Jing, Jiao Yuying research shows that the information used in mobile commerce context personalized recommendation system is a major trend in future studies, and as a basis for the establishment of a recommended learning resource model based on user context, user profile information for learning order resources[3].Yang Jun, who constructed a dynamic environment of dimensional user model, and "nearest neighbor" in the current context of the project score is calculated for the user to make a final recommendation to be recommended based on the user [4].Zhou Pu Xiong, who has this characteristic sensitivity context proposed "LBS+AR+Multi association rules, "the three-dimensional type recommended method for mobile information service, through the introduction of user context to improve the recommendation accuracy and quality[5].Deng Xiaoyi, who use the information to users clustering context, using social network theory and analyze the relationship between users and further improve the similarity calculation method, to further improve the quality of information recommended mobile commerce environment [6].

As can be seen above literature, domestic and foreign scholars on the recommendation based on the user profile information in question has made some achievements, most of the user's overall context information for the study, does not consider the information needs of different users in different context the impact of mobile commerce differences, lack of in-depth analysis of each user a personalized context. In this paper, according to the characteristics of mobile business environment, the elements of the context are classified in order to identify the main context factors affect mobile business users immediate information needs and through the training set way to find the largest impact on their information needs for each user $\mathrm{K}$ a context elements, based on this recommendation made into the mobile business model personalized context elements, finally, to improve the existing context-based multidimensional information recommendation algorithm, the personalized context as input conditions instead of the current context information, the project proposed context based on personalized recommendation algorithm recommended multidimensional information, the model to improve the mobile commerce recommended accuracy effectively improve the user experience provides a new way of thinking.

\section{Context and Personalized Mobile Commerce Environment Analysis}

\subsection{Context Meaning and Classification}

On the basis of A.K.Dey [7] and other scholars on the context described the connotation, combining features of mobile commerce, the context will be defined as immediate information needs of the time, location, environment and other information about the impact of mobile commerce is recommended, such as the user's preference and the at a time, location, weather conditions and so on.

According to the above definition, this paper impact of mobile instant messaging needs of business users to classify elements of the context:

(1)User context. User context mainly refers to behavioral state and its historical preference for basic user profile information and use the system when, for example: a user is studying or working, like sports or prefer to stay home when at rest, these context factors will affect the user's attention allocation and choice, is an important variable factors recommendation result.

(2)Location context. According to the literature[8]described the context known position, it refers to the user's location-related context information, such as the user is at home or at school, where the surrounding circumstances and information. User location information in 
this article include: User Area (city),the user's location type (school, home, shopping malls, supermarkets, entertainment),health workers around the user (students, family members, alone, colleagues) and the like.

(3)Time context. Time context mainly attribute to indicate the time in which the user profile and preferences, such as users browse through mobile devices time to buy the site(such as early ,in different time periods, night, etc.,.), holidays and weekdays user information resource different needs, like to focus on leisure and entertainment in the holidays, and on weekdays more attention to work and study class information.

(4)Environmental context. The environment here refers to environmental information users to use the system in which the recommendation, including the temperature, the weather and other natural environments. Mobile business user environment variable in the design of the system should recommended by environmental factors analysis to understand the true state of the user to use the system, found in favor of the needs of users and its changes.

(5)Equipment context. Currently, most mobile business users access to information resources through mobile phones, PAD and other mobile devices, including device information, one hardware device information, such as screen size, affect the amount of information per page two aspects; on the other hand is a network information, means of networking devices,including2G,3G,4G,and $\mathrm{Wi}-\mathrm{Fi}$, etc., different networking network speeds vary greatly, since the user is moving, it is recommended that the system needs time to capture this information in an appropriate manner the information recommended to the user, to provide users with better service.

\subsection{Personalized Context to Determine}

At present, the context recommended study context based mostly all of the user's overall context for the study, analysis was not performed for each user's personalization context, leading to the recommendation result to some extent deviate from the user immediate information needs. In the mobile business information recommendation process, context information vary, for example, tourism recommendation system in time, place, season and temperature are important elements of the context, but some people are sensitive elements of the above context, while others only the location or season-sensitive, and even some special groups, elements of these context have no impact on them .If you can perceive from all context information for each user to find their influence to make the final choice of context elements to be analyzed, not only can reduce the burden on the system is running, and make predictions more desirable[9].Such as Gao Min creatively put forward the concept of "personalized context", Zhou Pu Xiong learn from this thinking, the connotation of personalized context has been extended, we use the definition of Zhou Pu Xiong given, namely; personalized context refers to the impact instant information needs to be recommended to the $\mathrm{K}$ largest context elements [10].

In this paper, reference[10]approach to user rating, as measured by training on the training set Rating matrix elements of the recommended context to give added quality evaluation matrix, the $K$ best rating for each user under the $K$ context elements constituting the user's personalization context. Where in, $K$ is automatically set according to the optimal rating results. Personalized specific process context defined as follows:

The first step:The proportion of the user rating matrix according to the 80:20 is divided into training set $D$ and test set. Then $D$ according to the user's current profile $C=\left\{c_{1}, c_{2}, \ldots, c_{t}\right\}$ is divided into t subsets, namely $D=\left\{d_{1}, d_{2}, \ldots, d_{s}\right\}$.

Step Two: Let $P_{X(U, D)}$ recommended quality evaluation criteria, where $X$ is the recommendation algorithm is used, $U$ is a user set, $D$ is the training set. $P_{X\left(u_{s}, d_{s}\right)}$ represents the user $u_{s}$ to target customers, the training algorithm $X \quad c_{t}$ 
context-based training set $d_{s}$, users recommend $u_{s}$ for information quality rating.

The third step: the formation of user recommendations based on the evaluation matrix $P_{c}$ context:

$$
p_{c}=\left[\begin{array}{cccc}
p_{x\left(u_{1}, d_{1}\right)}, & p_{x\left(u_{1}, d_{2}\right)}, & \ldots, & p_{x\left(u_{1}, d_{s}\right)} \\
p_{x\left(u_{2}, d_{1}\right)}, & p_{x\left(u_{2}, d_{2}\right)}, & \ldots, & p_{x\left(u_{2}, d_{s}\right)} \\
\vdots & \vdots & & \vdots \\
p_{x\left(u_{s}, d_{1}\right)}, & p_{x\left(u_{s}, d_{2}\right)}, & \ldots, & p_{x\left(u_{s}, d_{s}\right)}
\end{array}\right]
$$

Step Four: According to $P_{c}$ for each user find the appropriate row of $K$ values corresponding to the optimum context composition elements of the context set $C^{\prime}$, that is, the user's personalization context.

\section{Construction of the Model}

Currently, context recommendation system based mostly in overall user context conducted on the basis of recommendations, ignoring the in-depth analysis for each user a personalized context, in view of this, the introduction of individual users in this article on the basis of the existing context studies context constructed mobile business model based on personalized recommendation context. Recommended process of the model begins to generate the user's personalized context via the user's current context and multidimensional historical rating data, and then used in combination obtained from a multidimensional rating data warehouse multidimensional rating data, obtained based on a personalized context $C^{\prime}$ multidimensional data $U($ User $) \times I($ Item $) \times C^{\prime}($ Personalized context $) \times R($ Rating $)$, and rating it as selecting the relevant data input conditions, based on these data is then selected based on rating personalized context multidimensional information recommendation algorithm to predict the user's project may rating, finally get to a user-supplied list of recommendations $\left\{I_{1}, I_{2}, I_{3}, \ldots\right\}$, model building framework As shown in Figure 1.

\subsection{Personalized Context Analysis Module}

Its main function is to identify all the context from the perceived information needs of the user instant messaging greatest impact context of $\mathrm{K}$ elements, namely the user's personalized profile, and users of the current context and multidimensional data warehouse is a system-generated rating of individual users the basis of the context. The user's current context, mobile devices can be built-in sensor, GPS, RFID and other means to obtain. Users multidimensional rating data warehouse to store the user has accepted the project multidimensional historical rating data, each multidimensional historical rating data first document by the user, item, context dimensions extract main properties of the dimensions of the document, combined with storage system rating data of components of a multi dimensional historical rating data and storage. Multidimensional rating data warehouse is an important basis for individual users to determine the context, as well as multidimensional information recommended key data sources.

\subsection{Multidimensional Information Recommended Module}

Multidimensional information recommendation is one of the most important components in the recommendation model of mobile commerce based on the personalized context. 
Multidimensional information recommended by the combined use of from multidimensional rating obtained in data warehouse multidimensional rating data and from personalized context analysis module to obtain users' personalized context, based on personalized context of multidimensional data $U \times I \times C^{\prime} \times R$, based on the use of the multidimensional information personalized context push the recommendation algorithm, the final recommendation and provide to the user.

\subsection{User and Recommendation System Interaction Module}

User and recommender system interaction module is an indispensable component of the recommended model. Users are mobile commerce recommendation system services, users to the multidimensional information recommendation module proposed recommendation request, system provides the recommendation results, user experience and recommended to rating results, user context information and user rating on the recommendation results immediately transmitted to the multidimensional rating data warehouse ,so as to realize the context with the rating update.

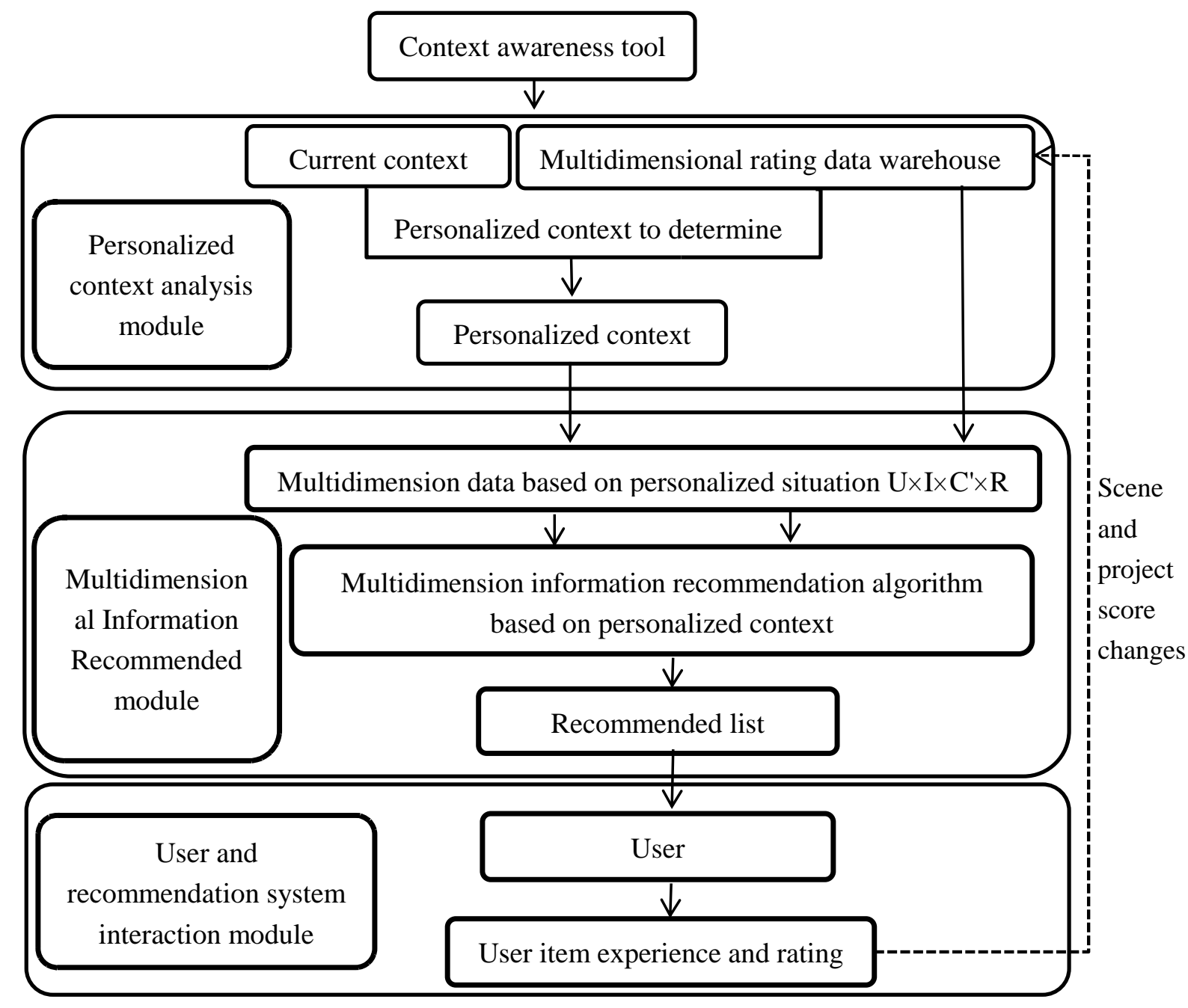

Figure 1. Recommendation Model of Mobile Commerce based on Personalized Context 


\section{Recommendation Model of Mobile Commerce based on Personalized Context}

\subsection{Algorithm Design Idea}

The mobile user's current personalized context $C^{\prime}$ instead of current context $C$ as a filter, excluding the user rating is less affected by context element contained in the rating data segment and selected contained in the context and the personalized context $C^{\prime}$ similar enough to rating data segment, and to determine the number according to the section of the existence of a user in different situations of the same item exists different rating, and then use the traditional two-dimensional recommendation algorithm were used to predict rating, the algorithm of the existing context based multidimensional information recommendation algorithm can not only alleviate the burden of system operation [11], and effectively improve the quality of recommendation.

Actual situation, the use of personalized context $C^{\prime}$ for multidimensional data selection, too explicit context elements may cause "thinning" of the data, so this use of selection criteria is $C^{\prime} \in S_{C^{\prime}}, S_{C^{\prime}}$ is a personalized context $C^{\prime}$ belongs to the superior context, for example $C^{\prime}=$ "girlfriend", $\mathrm{t}$ hen $S_{C^{\prime}}=$ "friend."

\subsection{Algorithm Process Description}

This section on the basis of the above models and design ideas, the algorithm of the input, output, the specific process of description.

Input:MR ( Multidimensional Ratings),MR $=\left\{\left(U_{j}, \quad, I_{k} C_{l}, R_{U_{j}, I_{k}, C_{l}}\right)\right\}$, $j=1, \ldots, m, k=1, \ldots, n$ (A total of $\mathrm{M}$ users, $\mathrm{N}$ items) $。 C_{l}$ is a multidimensional data rating of historical contexts, $l=1, \ldots, q$.

User current personalized context $C^{\prime}$, target user $u_{1}$

Output: the current personalized context $C^{\prime}$ target user $u_{1}$ on the item $I_{i}$ prediction rating $P_{u_{1}}, I_{i}, C^{\prime}$.

The specific process of the algorithm is divided into the following steps:

Step 1 On the multidimensional rating data filtering, select the context and the current personalized context $C^{\prime}$ with a similar enough rating data segment MD

Step 2 Step1 to judge whether due to data sparsity is difficult to construct contained in the context and the personalized context $C^{\prime}$ similar enough to rating data segment of MD, if the problem does not exist, direct the implementation of the next step. Otherwise, the $S_{C^{\prime}}$ instead of $C^{\prime}$ as the selection criteria for rating data segment selection, $S_{C^{\prime}}$ for a context $C^{\prime}$ is superior context.

Step $3 \mathrm{MD}$ to judge whether there is a user in different context of the same item exists different rating, if the problem does not exist, the direct execution of Step4.Otherwise, to the user in different context different rating on the same item for computation of aggregate, the algorithm by function AVG to compute the value of the average of these rating, the user of a item's final rating, namely: $R_{U_{j}}, I_{k}=A V G\left(U_{j}, I_{k}, C_{l}\right)$,so as to ensure the of the user with a project has only rating value, in order to get personalized context filtering after the final rating data segment of MD.

Step $4 \mathrm{MD}$ adopts the traditional two dimensional recommendation algorithm to calculate the similarity of the rating data segment. 
namely: $\operatorname{Sim}\left(u_{1}, U_{j}\right)=\frac{\sum_{s \in I\left(s_{u_{1}, U_{j}}\right)}\left(R_{u_{1}, s}-\overline{R_{u_{1}}}\right)\left(R_{U_{j}, s}-\overline{R_{U_{j}}}\right)}{\sqrt{\sum_{s \in S_{u_{1}, U_{j}}}\left(R_{u_{1}, s}-\overline{R_{u_{1}}}\right)^{2}} \sqrt{\sum_{s \in S_{u_{1}, U_{j}}}\left(R_{U_{j}, s}-\overline{R_{U_{j}}}\right)^{2}}}$

Among them, $\overline{R_{u_{1}}}$ is the average value of the target user $u_{1}$ for all items on the MD, $\overline{R_{U_{j}}}$ is the average value of the user $U_{j}$ in the for all items on the MD.

Step 5 To predict the rating of the items to be recommended.

namely: $\quad P_{u_{1}, I_{i}, c^{\prime}}=\overline{R_{u_{1}}}+k \sum_{j=1}^{N}\left(R_{U_{j}, I_{i}}-\overline{R_{U_{j}}}\right) \times \operatorname{sim}\left(u_{1}, U_{j}\right)$

Among them, $k=\frac{1}{\sum_{U_{j} \in U^{\prime}} \mid \operatorname{sim}\left(u_{1}, U_{j}\right)}, U^{\prime}$ is a collection of users $u_{1}$ with the most similar to the target user $\mathrm{N}$.The value of $\mathrm{N}$ is generally specified in advance by the recommender system.

Step 6 In descending order according to the rating value, and take out the top TOP-N as recommended in recommendation to target users.

\section{Experimental Results and Analysis}

\subsection{Experiment Data Sets and Evaluation Standard}

To verify the prediction effect of multidimensional information recommendation algorithm based on personalized context, we use Movie lens movie rating data set Minnesota University publicly available[12] and Books Review data set Book-Crossing provides numerical experiments[13],press 80:20 rating data will be randomly divided into a training set and a test set[14], and select the traditional method, the classic Slope One algorithm and context-based collaborative filtering algorithm were analyzed[15].

Lens Movie one hundred thousand data set published by the University of Minnesota in the United States contains 100000 users on 1700 films of 1000 records. Although this data set lack of external context information, but contains the user's gender, age, occupation and other user profile information, time context (such as time and holidays) through the data set can be indirectly time stamp data, and the data set has been cleared and quantization can be obtained indirectly, user preferences, it can be recommended algorithm directly. In addition, the data set for each user to provide a record rating at least 20 times, a certain ease data sparsity degree. Book-Crossing data set is 278858 user behavior information collected from the crawler Book-Crossing community of 271379 books were the book is used by Cai-Nicolas Ziegler. Contains the user ID, position, age, basic information and time context information (noon in the morning, afternoon, evening), the location context information (offices, homes, schools, restaurants), environmental context information (sound environment, space environment), state information (entertainment, work, learning) and other context information.

In this paper, the quality of recommended test method is widely used in the mean absolute error MAE[16]and $P(U) @ N[17]$ as a metric.

MAE by the deviation between the prediction rating and the actual rating is calculated to measure the accuracy of the results recommended, the smaller the deviation, the higher the accuracy of the prediction. MAE specific formula is: 


$$
M A E=\frac{\sum_{i=1}^{N}|p i-q i|}{N}
$$

Among them,the predicted user rating set to $\left\{p_{1}, p_{2} \ldots, p_{N}\right\}$,corresponding to the actual user rating set to $\left\{q_{1}, q_{2} \ldots, q_{n}\right\}$.

And $P(U) @ N$ represents the Top-N recommendation list is generated for the user $u$ ratio of the number of items that match their needs and $\mathrm{N}$, the formula is as follows:

$$
P(u) @ N=\frac{\text { relevant items in top } n \text { items for } u}{N}
$$

\subsection{Experimental Results and Analysis}

5.2.1. Personalized Context to Determine: Based on the concrete steps of (1) in 1.2 to train the user rating on the training set recommended evaluation matrix is obtained based on the context of the users:

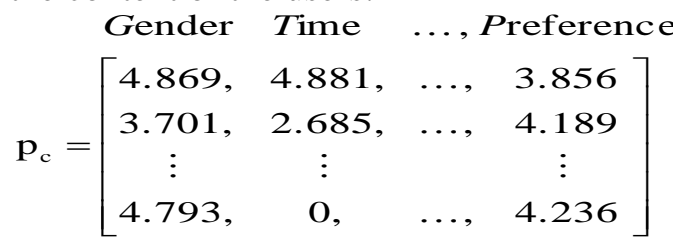

$$
p_{c}=\left[\begin{array}{cccc}
\text { Time } & \text { Location } & \ldots, & \text { Status } \\
3.758, & 4.782, & \ldots, & 2.957 \\
\vdots & 1.856, & \ldots, & 4.891 \\
2.973, & 4.579, & \ldots, & 3.632
\end{array}\right]
$$

Movie lens data set based on the context of the user Book-Crossing data set based on the context of the recommended evaluation matrix user recommended evaluation matrix.

Matrix rows represent individual users, the columns represent the corresponding context, each value represents the user $u_{s}$ under $c_{t}$ users of information recommendation quality rating, if the system setting $\mathrm{K}=5$, according to the rating level from all walks of life rating the highest five context composed of user's personalized context $C^{\prime}$.

5.2.2. Predicted Results Analysis: Using the proposed algorithm and the traditional algorithm, classic Slope One algorithm and the collaborative filtering algorithm based on context to compare, specific comparison results shown:

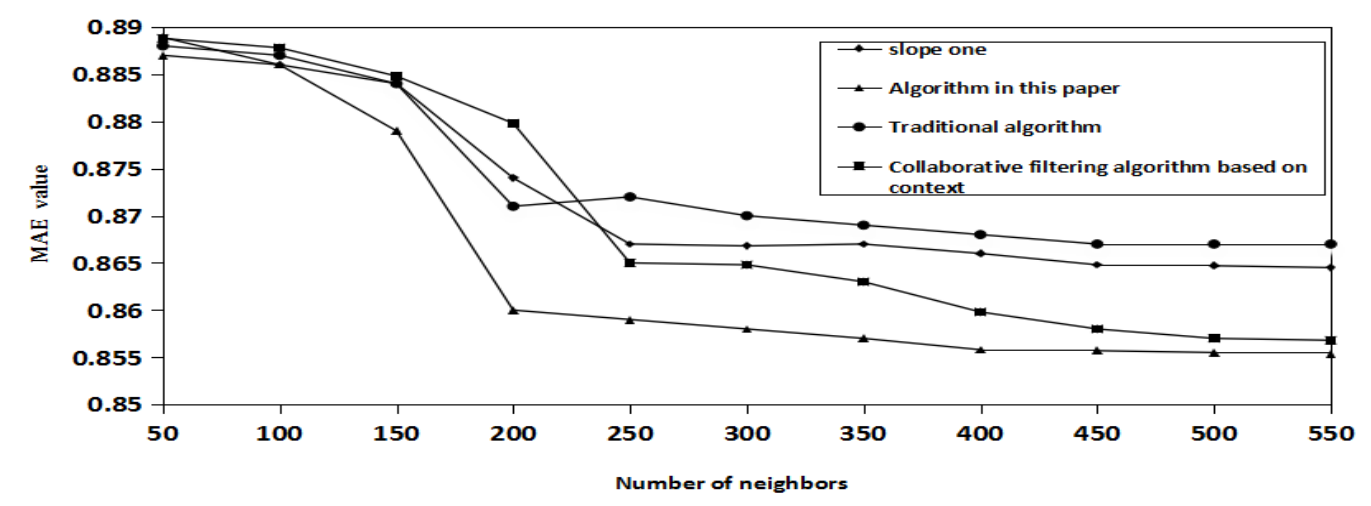

Figure 2. Four Kinds of Algorithms in Movie Lens Data Set of MAE Comparison Results 


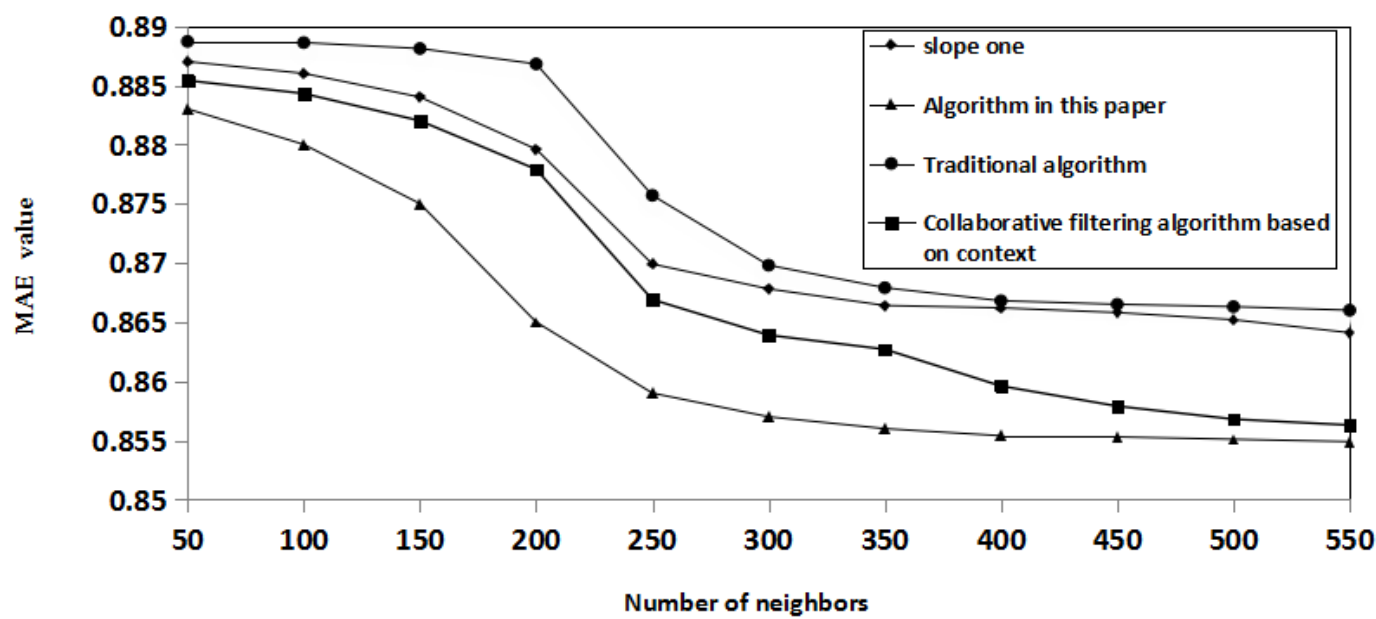
Figure 3. Four Kinds of Algorithms in Book-Crossing Data Set of MAE
Comparison Results

In Figure 2, because the movie lens data set lack part of the external context information, resulting in selected containing context and the current personalized context similarity ratings segment data is sparse, so that the value of the other three algorithms MAE volatile is larger,and less number of neighbor in the 0 150 neighbor users, four algorithms of MAE values were larger,but algorithm in this paper with the increase of the number of the neighbor, MAE value showed a faster decline trend and tended to be stable and the MAE value smaller than the other three algorithms.

In Figure 3, the four algorithms MAE value increases with the number of users neighbor showed a rapid decline, and the smallest MAE value of the algorithm in this paper, the highest prediction accuracy, which shows based on personalized context of multidimensional information recommendation algorithm is better than not considered or only consider the user's overall context information recommendation algorithm, because of the different context of the mobile business to different users' information needs of existence difference, and the result shows that, considering the effect of personalized context to the user can improve the recommendation quality.

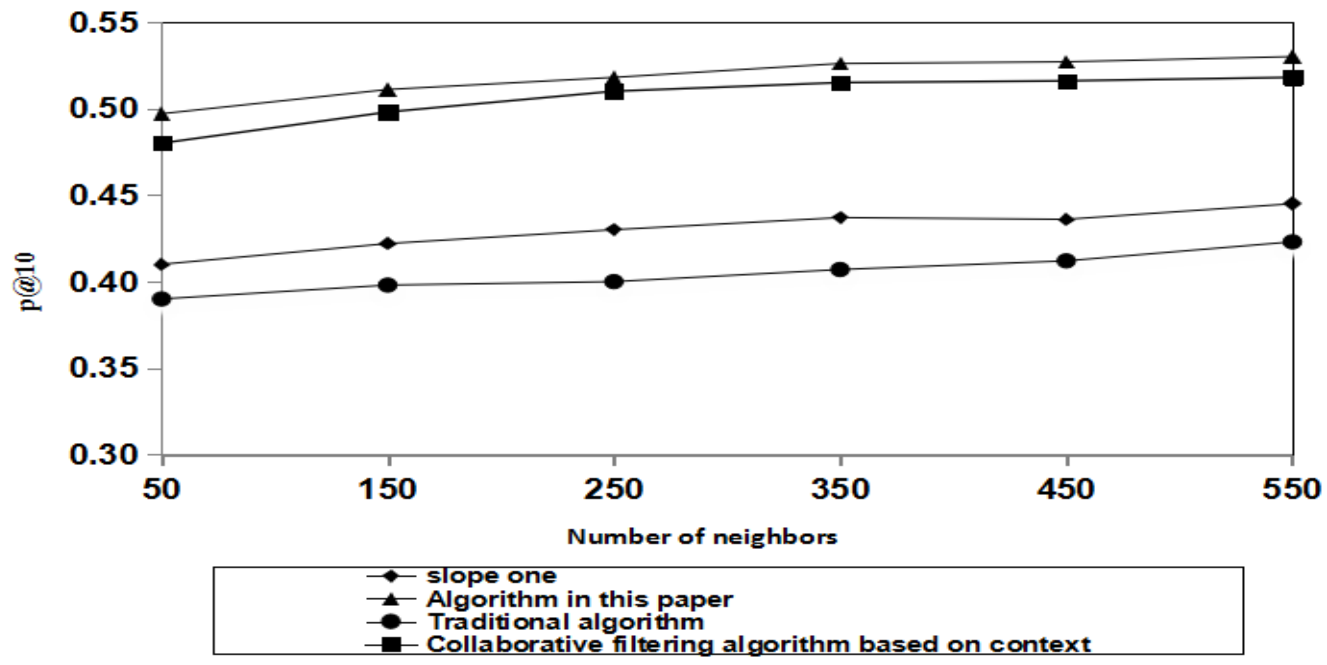

Figure 4. Four Kinds of Algorithms in Movie Lens Data Set of $P(U) @ N$ Comparison Results 


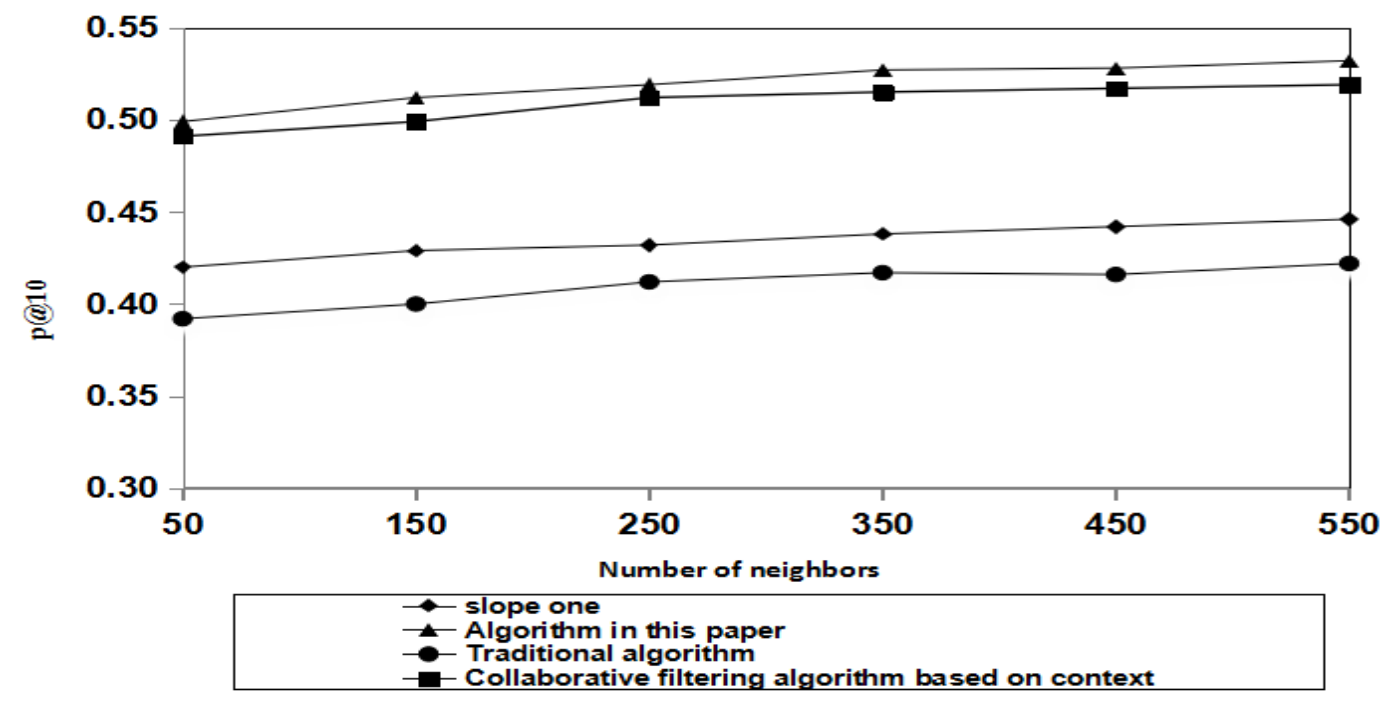

Figure 5. Four Kinds of Algorithms in Book-Crossing Data Set of $P(U) @ N$ Comparison Results

By Figure 4, and 5, can be seen in $P(U) @ N$ performance comparison,in the case of selecting a different number of neighbors, consider collaborative filtering algorithms overall context information is accurate than the high slope one algorithm and the traditional algorithm, in this paper based on personalized context of multidimensional information push recommendation algorithm effect more obvious, $P(U) @ N$ has a higher accuracy rate, it shows that the algorithm in this paper is reasonable to consider a personalized context elements, the higher the quality of recommendation, to better meet the needs of mobile business users instant information needs.

\section{Conclusion}

Based on existing research of context information recommendation, lack of in-depth analysis of the user personalization context, this paper proposes a model of mobile commerce recommendation based on personalized context, and improves the multidimensional information recommendation algorithm based on context. The model first calculate the each user's personalized context, and then use images of each user's personalized context recommendation to replace the current context of multidimensional information. Because the model to determine the user recommend most affected by context factors, more in line with the human the fact that there are different requirements in different context. Finally, in the Movie lens data set and the Book - Crossing data set, the results show that the proposed model and the improved algorithm can effectively improve rating prediction accuracy, further improve the quality of recommendation, more suitable for the personalized recommendation of mobile commerce environment problem. Future research work will combine social network analysis with the method of this paper to further improve the quality of recommendation algorithm.

\section{Acknowledgments}

This work was supported by National Natural Scientific Foundation (71272191),Research on knowledge transfer and sharing mechanism of IT Industry Alliance Based on cloud environment; Project of Natural Science Foundation of Heilongiang Province(G201301), Research on business model of mobile Cloud Computing Alliance in Heilongjiang Province; Philosophy and Social Sciences in Heilongjiang province to support the project(14E073),Research on service evolution mechanism and Realization Mechanism 
of equipment manufacturing industry in Heilongjiang Province.

\section{References}

[1] http://pema2011.cs.ucl.ac.uk/papers/pema2011_vico.pdf.

[2] T. H. Dao, S. R. Jeong and H. Ahn, "A novel recommendation model of location-based advertising:context-aware collabora-tive filtering using GA approach”, Expert Systems with Applications, vol. 39, no. 3, (2012), pp. 3731-3739.

[3] J. Yuan and Y. Y. Jiao, "Personalized recommendation of learning resources based on scene information", Information studies:Theory \& Application, vol. 7, (2009), pp. 116-119.

[4] J. Yang, H. L. Wang and D. X. Ai, "A new method of context-based multidimensional collaborative filtering recommendation", Library and Information Service, vol. 55, no. 21, (2011), pp. 110-111.

[5] P. X. Zhou and M. Y. Tao, "The Mobile Network Environment Situation Sensitive Personalized Information Recommendation System", Library and Information Service, vol. 56, no. 19, (2012), pp. 80-81.

[6] X. Y. Deng, C. Jin, Q. P. Han and K. L. Z. Tong, "Improved collaborative filtering model based on context clustering and user ranking", Systems Engineering-Theory\&Practice, vol. 33, no. 11, (2013), pp. 2945-2946.

[7] A. K. Dey, "Understanding and using context"Personal and Ubiquitous Computing Journal, vol. 5, no. 1, (2001), pp. 4-7.

[8] L. L. Zhai, J. Wang and X. Y. He, "The software industry virtual fleet cooperation competition mechanism", The front page.BEIJING:Science Press (2015).

[9] M. Gao and Z. F. Wu, "Personalized context and item based collaborative filtering recommendation", Journal of Southeast University:Natural Science Edition, (2009), pp. 29-30.

[10] P. X. Zhou, W. W. Xue and L. W. Zhao, "Research on Multi-Agent information recommendation based on personalized context”,Journal of Intelligence, (2015), pp. 182-183.

[11] J. Yang, Z. Mo, D. X. Ai and G. Q. Cai,"Research on multi dimension information recommendation based on dimension reduction of input scene",Theory \& Application, (2013), pp. 64-65.

[12] H. L. Xu, X. Wu and X. D. Li, "Comparison study of internet recommendation system",Journal of Software, vol. 20, no. 2, (2009), pp.350-362.

[13] Q. L. Gao, L. Gao, J. F. Yang and H. Wang, "A preference elicitation method based on users' cognitive behavior for context-aware recommender system",Chinese Journal of Computers, vol. 28, no. 9, (2015), pp. 1772-1774.

[14] X. Hu, X. W. Meng, Y. J. Zhang and Y. C. Shi,"Recommendation Algorithm Combing Item Features and Trust Relationship of Mobile Users", Journal of Software, vol. 25, no. 8, (2014), pp.1824-1825.

[15] L. L. Zhai, Y. F. Liu, J. Wang and N. N. Li, "Software industry virtual cluster inter firm trust evolution game research", Chinese Journal of Management Science, vol. 22, no. 12, (2014), pp. 122-123.

[16] J. Kim, D. Lee and K.Chung, "Item Recommendation Based On Context-Aware Model for Service", Multimedia Tools and Applications, vol. 71, no. 2, (2014), pp. 855-872.

[17] R. Cyohulyad and P. Leray, "A Personalized Recommender System Relational Model and Users' Preferences”, Procedia Computer Science, (2014), pp. 1063-1067. 
International Journal of $u$ - and e- Service, Science and Technology Vol. 10, No. 1 (2017) 